\section{Preparation of End-Grafted Polyelectrolyte Brushes on Nanoscale Probe Tips Using an Electric Field}

\author{
J oonil Seog, † Delphine M. Dean,‡ Eliot H. Frank, \\ Christine Ortiz, $\$$ and Alan J . Grodzinsky*,t,‡
}

Departments of Mechanical Engineering, Electrical Engineering and Computer Science, and Materials Science and Engineering, Massachusetts I nstitute of Technol ogy, 77 Massachusetts Avenue, Cambridge, Massachusetts 02139

Received August 19, 2003

Revised Manuscript Received November 12, 2003

\section{Introduction}

The atomic force microscope (AFM) and related nanomechanical instruments have become fundamental tools for studying molecular, colloidal, and surface forces in physiological fluid environments down to the $\mathrm{pN}$ range. ${ }^{1-5}$ A key component of the AFM is the soft microfabricated cantilever force transducer and fine probe tip (with a typical end radius of $R_{\text {TIP }}<50 \mathrm{~nm}$ ) that deflects when interacting with a sample surface. Standard $\mathrm{Si}_{3} \mathrm{~N}_{4}$ probe tips have been used for studying the nanomechanical properties of macromolecules on surfaces, ${ }^{1}$ and probe tips functionalized with proteins, ligands and receptors, cells, and nanotubes have enabled studies of biologically relevant intermolecular interactions. ${ }^{2-5}$ Probe tip functionalization has been achieved by covalent immobilization, nonspecific physisorption, and conventional adhesives for larger structures $(\geq 1$ $\mu \mathrm{m}) .{ }^{6-10}$ However, attachment of macromolecules to the apex of a probe tip with a prespecified orientation, conformation, and density is difficult due to the small surface area involved and, for polyelectrolytes, the presence of fixed charge groups. Functionalization and subsequent characterization of parameters such as the chain grafting density, $\Gamma$ (chains $/ \mathrm{nm}^{2}$ ), in the vicinity of the tip apex are also difficult and critical to the interpretation of nanomechanical data relating interaction force to tip-sample separation distance.

Our recent studies of the biological polyelectrolyte molecules in cartilage, ${ }^{11}$ chondroitin sulfate glycosaminoglycans (GAGs), called for a new method of attachment and assessment on nanoscale probe tips in order to carry out nanomechanical experiments between two opposing, end-grafted GAG brushes which more closely mimic the physiological conformations found in native tissue. GAGs were chemically end-grafted to an Aucoated $\mathrm{Si}_{3} \mathrm{~N}_{4}$ probe tip by means of an electric field applied between the probe tip and a nearby Pt electrode. Previously, an el ectric fiel d was used to attract charged DNA oligonucleotides to a monolayer of single-stranded DNA that had been immobilized on an Au-coated sensor surface through a Au-thiol attachment and a $300 \mathrm{mV}$ potential between the sensor and a Pt electrode was found to enhance hybridization of the DNA oligonucleotides to the DNA monolayer. ${ }^{13}$ We extended this

\footnotetext{
* Corresponding author. E-mail: alg@mit.edu.

† Departments of Mechanical Engineering, Massachusetts Institute of Technology.

₹ Electrical Engineering and Computer Science, Massachusetts Institute of Technology.

$\S$ Materials Science and Engineering, Massachusetts Institute of Technology.
}

methodology to use an electric field to drive charged GAG molecules to a nanosized probe tip, thus increasing the local polyel ectrolyte concentration in the vicinity of the probe tip and chemisorption via an end-terminal functionality.

\section{Experimental Section}

Methods described previously ${ }^{11}$ for chemically end-grafting mono(thiol)-terminated chondroitin sulfate GAGs to Au-coated Si planar substrates were adapted for grafting GAGs to Aucoated $\mathrm{Si}_{3} \mathrm{~N}_{4}$ probe tips at the end of a soft cantilever (Thermomicroscopes, Inc., spring constant $=0.01 \mathrm{~N} / \mathrm{m}$ ). "Passive functionalization" involved immersing Au-coated probe tips into phosphate buffer solution (PBS, $\mathrm{pH}=7.4$, ionic strength, IS $=0.15 \mathrm{M}$ ) containing $1 \mathrm{mg} / \mathrm{mL}$ mono(thiol)-terminated CSGAG for $9 \mathrm{~h}$. After reaction, the probe tips were immersed in $5 \mathrm{mM}$ 11-mercaptoundecanol, $\mathrm{HS}\left(\mathrm{CH}_{2}\right)_{11} \mathrm{OH}$, ethanol solutions for 15 min to "backfill" the unreacted portions of the surface. "Active functionalization" involved applying an electric field for $9 \mathrm{~h}$ between the probe tip and a Pt electrode immersed in $1 \mathrm{mg} / \mathrm{mL}$ GAG PBS solution in the closed liquid cell of an AFM (Multimode IIIA, Digital Instruments, Santa Barbara, CA). The probe tip was grounded, and a negative voltage $(-0.15$ V) was applied to the Pt cathode via a cap on the piezoel ectric scanner (Figure 1a).

The separation distance between the probe tip and the Pt electrode was approximately $100 \mu \mathrm{m}$. The sharp probe tip geometry resulted in a 10-fold higher E-field strength near the tip apex ( $3000 \mathrm{~V} / \mathrm{m})$ compared to voltage near the Pt surface (F igure 1b). Figure 1c is the current-voltage curve for thetip$\mathrm{Pt}$ system which shows that at the low voltage used here $(<|0.15| \mathrm{V})$, the resulting small, non-Faradaic current $(<200$ $\mathrm{nA}$ ) minimizes chemical reactions at the probe tip that could lead to electrolysis or disrupt the CS-GAG end-grafting chemistry. The actively modified probetip was backfilled using the same procedure as for the passive functionalization method.

Planar $\mathrm{OH}$-terminated self-assembling monolayer (SAM) substrates were prepared on Au-coated Si by immersing in a $1 \mathrm{mM}$ solution of 11-mercaptoundecanol for $24 \mathrm{~h}$. Nanomechanical measurements were performed using a Molecular Force Probe (MFP) (Asylum Research, Santa Barbara, CA) to measure force, F, vs tip-substrate separation distance, $D$, at a constant z-piezo displacement rate of $1 \mu \mathrm{m} / \mathrm{s}$ in $\mathrm{NaCl}$ aqueous solutions at room temperature. A full description of this instrument, its limit of force and displacement detection in fluids, and details of measurement errors, were given previously. ${ }^{11} \mathrm{Nanomechanical} \mathrm{data} \mathrm{presented} \mathrm{are} \mathrm{averaged} \mathrm{curves}$ of 10-15 individual experiments, on approach of the probe tip normal to the sample surface, at a minimum of three different sample locations and have a standard deviation of $<20 \mathrm{pN}$. The linear constant compliance region corresponding to contact with the underlying Au substrate was clearly observed and set to $\mathrm{D}=0$.

\section{Results and Discussion}

Figure 2 compares average force/radius vs distance profiles on approach for the actively and passively functionalized GAG probe tips vs an $\mathrm{OH}$-terminated SAM planar substrate in $0.1 \mathrm{M} \mathrm{NaCl}, \mathrm{pH}$ 5.6. Both probe tips showed a nonlinear, purely repulsive interaction force for $D<40 \mathrm{~nm}$ with no attractive jump-tocontact. The electrically functionalized probe tip produced a significantly higher force over a longer distance range, suggesting the presence of a higher density of charged GAGs on the probe tip. Since the electrical Debye length, $\kappa^{-1}$, is $1 \mathrm{~nm}$ at $0.1 \mathrm{M}$ ionic strength (IS) surface anion adsorption to the probe tip, al one, could 
(a)

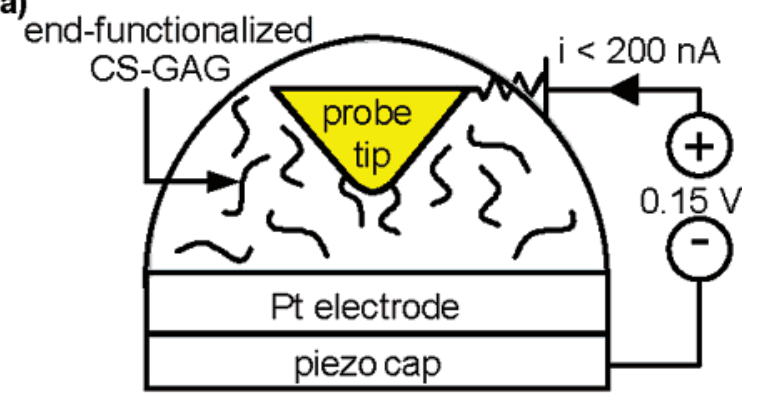

(b)

(c)
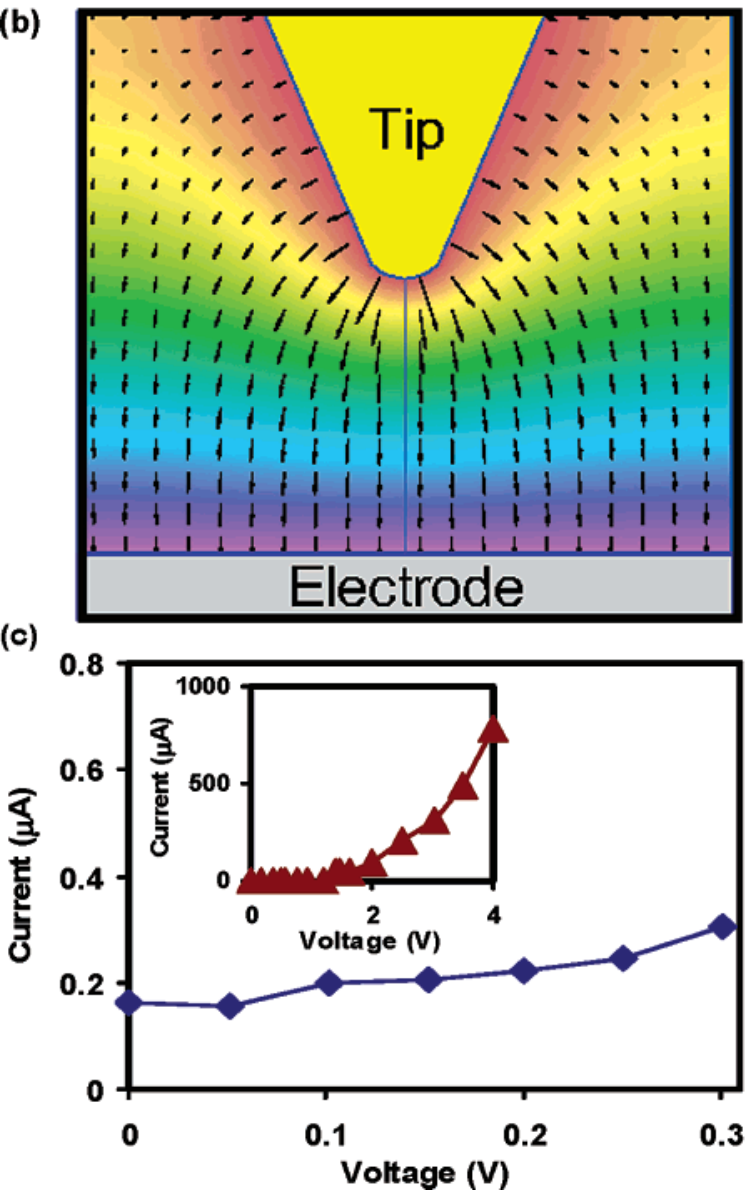

Figure 1. (a) End-grafting GAGs to a nanosized probe tip using an electric field, (b) 2-D map of E-field lines between probe tip and electrode calculated using QuickField finite element solver (DK-5700, Svendborg, Denmark), where arrow length and direction correspond to E-field magnitude and direction, and (c) current vs voltage across probe tip in $0.1 \mathrm{M}$ $\mathrm{NaCl}, \mathrm{pH}=5.6$ aqueous solution (inset displays expanded scales).

not account for the long distance ranges of the interaction, which is expected to start around $5 \mathrm{~nm}\left(\sim 5 \kappa^{-1}\right) .{ }^{13}$ To estimate the GAG grafting density on the probe tip obtained by electrical functionalization, the data of Figure 2 were compared to the predictions of a PoissonBoltzmann based charged rod model (solid lines) for the el ectrostatic double layer repulsion force. ${ }^{14}$ The $\mathrm{OH}$ terminated SAM was represented as a neutral planar substrate and the GAG functionalized probe tip was represented as a hemisphere with end-grafted, charged, cylindrical rods of finite height with uniform fixed volume charge density. The parameters that were fixed in the model included the polyelectrolyte brush height ( $h=45 \mathrm{~nm}$, set to the distance at which the force increased above the noise minus $\left.5 \kappa^{-1}\right)$, the rod radius $(r=2 \mathrm{~nm})$, and the known charge per GAG chain $(\mathrm{Q}=$

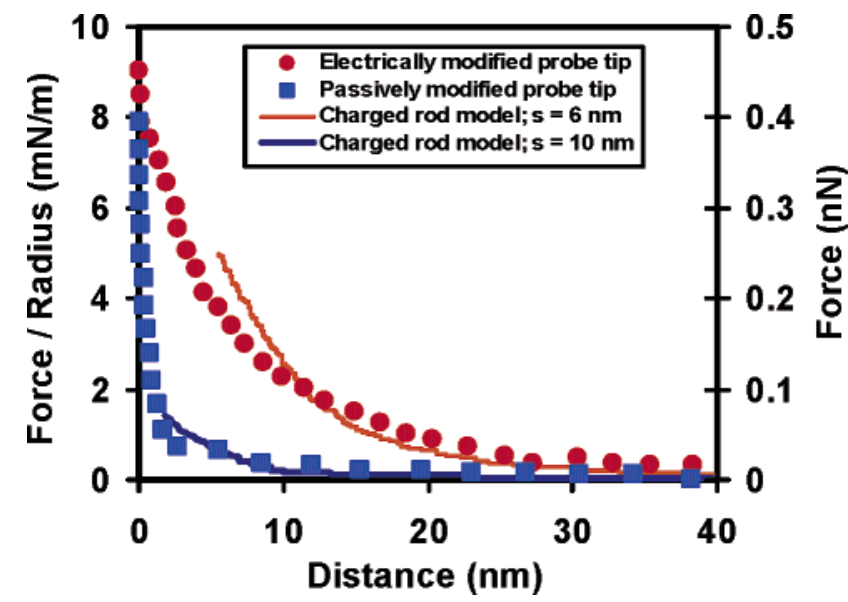

Figure 2. Nanomechanical data for actively and passively functionalized GAG probe tips vs $\mathrm{OH}$-terminated SAM planar substrate compared to theoretical models (IS $=0.1 \mathrm{M}$, $\mathrm{pH}=5.6)$.

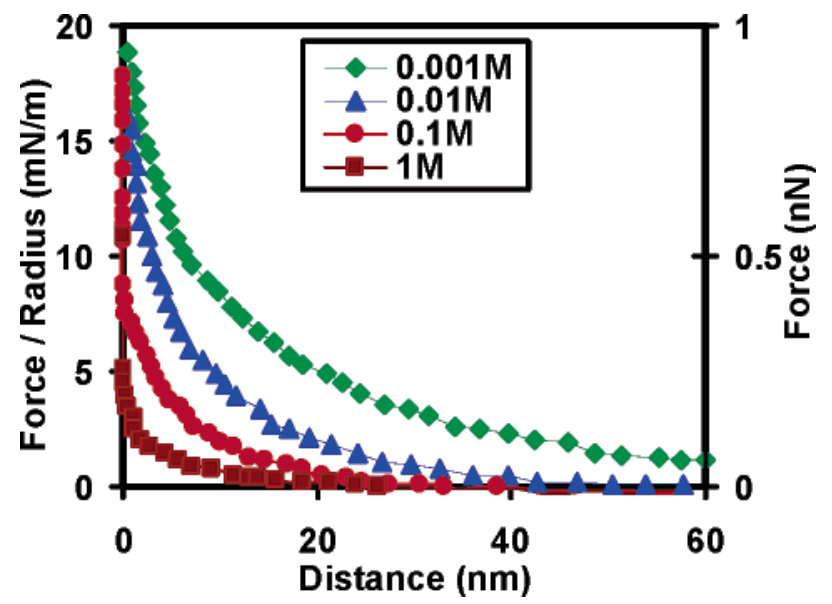

Figure 3. Nanomechanical data for actively functionalized GAG probe tip vs OH-terminated SAM substrate at various IS, $\mathrm{pH}=5.6$

$-8.0 \times 10^{-18} \mathrm{C}$ ). The adjustable parameters in the model were the distance between neighboring GAG chains (s) and $R_{\text {TIP. }}$. The nanomechanical data was fit between $D=10-80 \mathrm{~nm}$ using the method of least squares. The resulting values were $s=10 \mathrm{~nm}, \Gamma=0.01$ chains $/ \mathrm{nm}^{2}$ for the passively modified probe tip and $\mathrm{s}=$ $6 \mathrm{~nm}, \Gamma=0.028$ chains $/ \mathrm{nm}^{2}$ for the actively modified probe tip with $R_{T I P}=50 \mathrm{~nm}$, thus showing that the E-field gave a $\sim 3$-fold increase in $\Gamma$. $\Gamma$ obtained via electrical functionalization corresponds well with values obtained on metabolically radiolabeled GAGs attached to planar substrates using the same chemical reaction conditions and measured via the technique of scintillation counting. ${ }^{11}$

Figure 3 shows the average force/radius and force vs distance profiles for the actively functionalized GAG probe tip vs an $\mathrm{OH}$-terminated SAM planar substrate on approach at various IS and $\mathrm{pH} \sim 5$.6. The repulsive force was observed to decrease in magnitude and range with increasing IS, consistent with the well-known effect of salt screening of the electrostatic double layer forces.

In addition, the observed decrease in HRFS-measured force when the $\mathrm{pH}$ was decreased from 7 to 3 (Figure 4, IS $=0.015 \mathrm{M})$ is consistent with the fact that both $\mathrm{SO}_{3}{ }^{-}$ and $\mathrm{COO}^{-}$groups on the GAG are charged at $\mathrm{pH} 7$ (the $\mathrm{pK}_{\mathrm{a}}$ of the $\mathrm{SO}_{3}{ }^{-}$group is $2-2.5^{15}$ and that of the $\mathrm{COO}^{-}$ group is $3.5-4^{16}$ ) whereas the $\mathrm{SO}_{3}{ }^{-}$group remains 


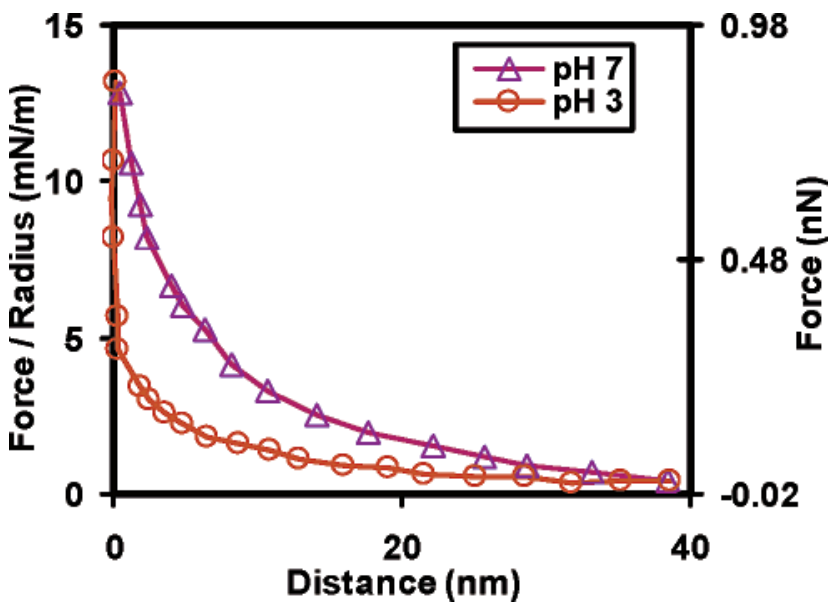

Figure 4. Nanomechanical data for actively functionalized CS-GAG probe tip vs $\mathrm{OH}$-terminated SAM substrate at $\mathrm{pH} 7$ and $3(\mathrm{IS}=0.015 \mathrm{M})$.

charged and most $\mathrm{COOH}$ groups are protonated at $\mathrm{pH}$ 3 , leading to about $50 \%$ reduction in the fixed charge.

To our knowledge, this is the first report of the use of an electric field to enable end-grafting of charged polyelectrolytes to a nanosized probe tip to study intermolecular interactions. The sharp tip geometry amplified the E-field strength near the tip apex which, in turn, enhanced migration of charged GAGs to the probe tip while simultaneously allowing a small total voltage drop and non-F aradaic total current. The presence of GAG on the probe tip was verified by a distinct increase in the magnitude of the repulsive force, measurements at varying $\mathrm{pH}$ and IS in conjunction with a Poisson-Boltzmann based theoretical model to approximate $\Gamma$. Wefeel that the methodology reported here holds promise since direct chemical characterization techniques on such a small surface area of hemispherical and variable geometry containing a small number of molecules is difficult, if not impossible, at this time.
Acknowledgment. The authors thank A. H. K. Plaas and S. Wong-Palms for providing GAGs and P. Laibinis for insightful advice and providing 11-mercaptoundecanol. This work was supported by the DupontMIT Alliance, NIH Grant AR45779, and a Whitaker Foundation graduate fellowship (D.M.D.).

\section{References and Notes}

(1) Ortiz, C.; Hadziioannou, G. Macromolecules 1999, 32, 780787.

(2) Florin, E. L.; Moy, V. T.; Gaub, H. E. Science 1994, 264, 415-417.

(3) Lower, S. K.; Hochella, M. F., J r.; Beveridge, T. J . Science 2001, 292, 1360-1363.

(4) Hafner, J . H.; Cheung, C. L.; Woolley, A. T.; Lieber, C. M. Prog. Biophys. Mol. Biol. 2001, 77, 73-110.

(5) Heinz, W. F.; Hoh, J. H. Trends Biotech. 1999, 17, 143150.

(6) Strunz, T.; Oroszlan, K.; Schafer, R.; Guntherodt, H. J . Proc. Natl. Acad. Sci. U.S.A. 1999, 96, 11277-11282.

(7) McKendry, R.; Theoclitou, M. E.; Rayment, T.; Abell, C. Nature (London) 1998, 391, 566-568.

(8) Hugel, T.; Holland, N. B.; Cattani, A.; Moroder, L.; Seitz, M.; Gaub, H. E. Science 2002, 296, 1103-1106.

(9) Rief, M.; Oesterhelt, F.; Heymann, B.; Gaub, H. E. Science 1997, 275, 1295-1297.

(10) Ducker, W. A.; Senden, T. J .; Pashley, R. M. Nature(London) 1991, 353, 239-241.

(11) Seog, J .; Dean, D.; Plaas, A. H. K.; Wong-Palms, S.; Grodzinsky, A. J .; Ortiz, C. Macromol ecules 2002, 35, 56015615.

(12) Heaton, R. J .; Peterson, A. W.; Georgiadis, R. M. Proc. Natl. Acad. Sci. U.S.A. 2001, 98, 3701-3704.

(13) Biggs, S.; Mulvaney, P.; Zukoski, C. F.; Grieser, F. J . Am. Chem. Soc. 1994, 116, 9150-9157.

(14) Dean, D.; Seog, J .; Ortiz, C.; Grodzinsky, A. J . Langmuir 2003, 19, 5526-5539.

(15) Kuettner, K.; Lindenbaum, A. Biochim. Biophys. Acta 1965, $101,223-225$

(16) Freeman, W. D. S. C.; Maroudas, A. Ann. Rheum. Dis. 1975 $34,44-45$

MA0352274 